\title{
Progress on the infrared structure of multi-particle gauge theory amplitudes
}

\section{Lorenzo Magnea*}

University of Torino and INFN, Sezione di Torino

E-mail: lorenzo.magnea@unito.it

\begin{abstract}
I will review some of the recent intense activity concerning infrared and collinear divergences in gauge theory amplitudes. The central quantity in these studies is the multi-particle soft anomalous dimension matrix, which is completely known at two loops for both massless and massive particles, and whose properties are currently being studied at three-loops and beyond. I will describe how, in the massless case, the simple dipole-like structure of the anomalous dimension up to two loops can be exploited in the high-energy limit to study effects that go beyond the standard form of Regge factorization. Furthermore, I will briefly review some of the techniques that have recently been developed to compute the soft anomalous dimension at high orders in perturbation theory, and I will give some examples of applications, including a result valid to all orders in perturbation theory for a specific class of diagrams.
\end{abstract}

Loops and Legs in Quantum Field Theory - LL 2014,

27 April - 2 May 2014

Weimar, Germany

\footnotetext{
* Speaker.
} 


\section{Introduction}

The interest in the structure of infrared divergences of non-abelian gauge theory amplitudes has revived in recent years, due to several reasons, both phenomenological and theoretical.

A practical concern is the fact that infrared singularities must be understood in great detail in order to construct efficient subtraction algorithms, which are needed in order to compute infraredsafe quantities at non-trivial orders in perturbation theory. The need to provide precise predictions for complex processes such as jet production at LHC has driven a vast effort to push these calculations beyond NLO [1], focusing interest on the infrared structure of scattering amplitudes at high orders.

Another issue of great relevance for phenomenology is the need to resum large logarithmic corrections, which jeopardize the reliability of the perturbative expansion for many cross sections of physical interest. The technology of resummation is based upon the knowledge of soft and collinear singularities [2,3]: indeed, while actual divergences cancel in physical cross sections, they leave behind logarithmic enhancements, which can be computed to all orders in perturbation theory with increasing accuracy, provided the structure of singularities is sufficiently well understood. Resummations are well developed for cross sections involving only two colored particles at tree level, such as electroweak vector boson production, DIS, or Higgs production [4]. The needs of LHC have however generated interest in the possibility to provide resummed predictions for more complicated multi-particle processes. These predictions in turn require a deeper understanding of infrared divergences of multi-particle amplitudes.

Finally, it must be noted that the study of long-distance singularities is of great interest also from a purely theoretical point of view. One aspect of this interest is the role played by singularities in the scattering amplitudes of $N=4$ Super Yang-Mills theory, which have been the focus of massive investigations and impressive progress in the past several years [5]. In this case the quantum (super) conformal invariance of the theory makes the very idea of an $S$-matrix rather precarious: indeed, scattering amplitudes are, to a large extent, defined by their infrared regularization, and infrared divergences in fact determine the entire structure of the perturbative expansion, at least up to the conformally invariant 'remainder functions' which start arising at two-loops and with at least six colored particles [6,7]. The all-order knowledge of infrared divergences for planar amplitudes has been instrumental to provide ideas and check results in this field [8], and it is to be hoped that the same will happen in the non-planar case as well.

More generally, infrared singularities provide a window into the all-order behavior of perturbative gauge theories: they are sufficiently simple and universal to be computable with great accuracy, yet they retain highly nontrivial information about dynamics. Multi-particle amplitudes are especially interesting in this regard, since they display, at the level of the anomalous dimension, highly non-trivial correlations between color and kinematic degrees of freedom, which must ultimately be reflected (in QCD) by the patterns of parton evolution which prepare colored particles for hadronization.

In what follows, I will focus mostly on progress which has been achieved in the last couple of years on two fronts: on the one hand, the application of our understanding of infrared structure to the high-energy limit, where one can control contributions going beyond the simplest form of Reggeization [9, 10], making predictions at two and three loops [11]; on the other hand, the devel- 
opment and application of computational tools for the calculation of the soft anomalous dimension at high orders [12,13], and in particular at three loops. This calculation, which would have looked unfeasible just a few years ago, appears now within reach.

\section{On the structure of infrared divergences}

The distinguishing character of soft and collinear radiation is universality. Divergences arise from emissions that are unsuppressed at large distances and long times, and thus happen on energy scales which are drastically different from those associated with hard processes. In other words, infrared radiation does not possess the resolving power to probe the hard scattering: it happens 'much later' or 'much earlier', so that quantum-mechanical interference with the short-distance process is suppressed by powers of the hard scale. While this picture is rather intuitive and physically appealing, the structure of perturbation theory makes proving it very difficult $[2,14,15]$. When the dust settles, one is left with a simple factorization formula, which applies to all gauge theory amplitudes, and is exact for infrared divergent contributions. One writes $[16,17]$

$$
\mathscr{M}\left(\frac{p_{i}}{\mu}, \alpha_{s}\right)=\mathscr{Z}\left(\frac{p_{i}}{\mu}, \alpha_{s}\right) \mathscr{H}\left(\frac{p_{i}}{\mu}, \alpha_{s}\right)
$$

where the scattering amplitude $\mathscr{M}$, involving $n$ colored particles with momenta $p_{i}$, is a vector in color space, while $\mathscr{Z}$ is a universal operator encoding infrared divergences, and acting upon the finite vector of "matching coefficients" $\mathscr{H}$. The infrared operator $\mathscr{Z}$ obeys a renormalization group equation which, in dimensional regularization [18] and for $d=4-2 \varepsilon>4$, has a very simple solution, expressed in terms of an anomalous dimension matrix $\Gamma$ as

$$
\mathscr{Z}\left(\frac{p_{i}}{\mu}, \alpha_{s}\right)=\mathscr{P} \exp \left[\frac{1}{2} \int_{0}^{\mu^{2}} \frac{d \lambda^{2}}{\lambda^{2}} \Gamma\left(\frac{p_{i}}{\lambda}, \alpha_{s}\left(\lambda^{2}\right)\right)\right] .
$$

Quite naturally, the matrix $\Gamma$ is the focus of all investigations in this field: its knowledge completely solves the perturbative infrared problem for all gauge theory scattering amplitudes. Currently, $\Gamma$ is known at two loops, both for massless [19, 20] and for massive [21, 22, 23, 24, 25] particles. In the massless case, the problem has an extra symmetry, since the momenta of all hard particles can be independently rescaled without affecting infrared emissions. This symmetry puts strong constraints on the soft anomalous dimension matrix: a simple solution to these constraints is the dipole formula [26, 27]

$$
\Gamma_{\text {dip }}\left(\frac{p_{i}}{\lambda}, \alpha_{S}\left(\lambda^{2}\right)\right)=\frac{1}{4} \widehat{\gamma}_{K}\left(\alpha_{S}\left(\lambda^{2}\right)\right) \sum_{(i, j)} \ln \left(\frac{-s_{i j}}{\lambda^{2}}\right) \mathbf{T}_{i} \cdot \mathbf{T}_{j}-\sum_{i} \gamma_{i}\left(\alpha_{S}\left(\lambda^{2}\right)\right)
$$

stating that only two-particle color and kinematic correlations contribute to the soft anomalous dimension. In Eq. (2.3) $\mathbf{T}_{i}$ is a color generator in the representation of particle $i$, and the running coupling enters only through the anomalous dimensions $\gamma_{i}$, responsible for collinear emissions from hard parton $i$, and $\widehat{\gamma}_{K}$, which is the (light-like) cusp anomalous dimension [28, 29], rescaled by the quadratic Casimir eigenvalue of the relevant color representation. As a consequence, so long as Eq. (2.3) applies, the path ordering operator $\mathscr{P}$ can be omitted in Eq. (2.2). The dipole formula 
is exact at two loops, and it is known that it can only receive corrections starting with three-loop four-point amplitudes, due to the existence at that order of conformally invariant cross-ratios of momenta which automatically satisfy the rescaling constraints. Evidence for the existence of such corrections, albeit at four loops, was recently uncovered in Ref. [30]. Further corrections could arise, starting at four loops, if the cusp anomalous dimension $\gamma_{K}\left(\alpha_{s}\right)$ were to contain terms proportional to higher rank Casimir eigenvalues, at that order or beyond. The nature of the corrections to the dipole formula is currently the subject of intense investigations [31, 32].

For massive particles, the simplicity arising from the extra rescaling symmetry is lost, and indeed the two-loop calculation shows that tripole correlations among colored particles do arise. Denoting the Minkowsky angle between hard particles $i$ and $j$ by

$$
\gamma_{i j}=\frac{2 p_{i} \cdot p_{j}}{\sqrt{p_{i}^{2} p_{j}^{2}}} \equiv-\alpha_{i j}-\frac{1}{\alpha_{i j}}
$$

and then defining $\xi_{i j} \equiv \log \alpha_{i j}$, one can express three-particle correlations in the two-loop soft anomalous dimension as

$$
\Gamma_{\text {trip }}^{(2)}\left(\xi_{m n}\right)=\mathrm{i} f_{a b c} \sum_{i j k} \mathbf{T}_{i}^{a} \mathbf{T}_{j}^{b} \mathbf{T}_{k}^{c} \mathscr{F}^{(2)}\left(\xi_{i j}, \xi_{j k}, \xi_{k i}\right)
$$

where [22]

$$
\mathscr{F}^{(2)}\left(\xi_{i j}, \xi_{j k}, \xi_{k i}\right)=\frac{4}{3} \sum_{i j k} \varepsilon_{i j k} g\left(\xi_{i j}\right) \xi_{j k} \operatorname{coth}\left(\xi_{j k}\right)
$$

Remarkably, Eq. (2.6) has a factorized form in terms of relatively simple functions of individual cusp angles: indeed, $g$ is a function of uniform weight $w=2$, while the factor $\xi_{i j} \operatorname{coth} \xi_{i j}$ is proportional to the one-loop non-light-like cusp anomalous dimension. Clearly, also in the massive case, there is a structural simplicity which has yet to be fully understood.

\section{On the high-energy limit}

As a first example of application of our understanding of long-distance singularities, let's consider the high-energy limit. Focusing on four-point amplitudes, this is the limit in which $s \rightarrow \infty$, while $|t|$ remains limited. To the extent that the dipole formula, Eq. (2.3), applies, the infrared operator $\mathscr{Z}$ in this limit simplifies considerably, taking on the form $[9,10,11,33]$

$$
\mathscr{Z}\left(\frac{s}{\mu^{2}}, \frac{t}{\mu^{2}}, \alpha_{s}\right)=\mathscr{Z}_{1, \mathbf{R}}\left(\frac{t}{\mu^{2}}, \alpha_{s}\right) \widetilde{\mathscr{Z}_{S}}\left(\frac{s}{t}, \alpha_{s}\right)+\mathscr{O}\left(\frac{t}{s}\right),
$$

where $\widetilde{\mathscr{Z}_{S}}$ is a matrix carrying all the leading-power energy dependence, while $\mathscr{Z}_{1, \mathbf{R}}$ is a colorsinglet factor. Introducing 'Mandelstam' combinations of color generators, $\mathbf{T}_{s} \equiv \mathbf{T}_{1}+\mathbf{T}_{2}$, and similarly for $\mathbf{T}_{t}$ and $\mathbf{T}_{u}$, one can write the operator $\widetilde{\mathscr{Z}_{S}}$ as

$$
\widetilde{\mathscr{Z}_{\mathrm{S}}}\left(\frac{s}{t}, \alpha_{s}\right)=\exp \left\{K\left(\alpha_{s}\right)\left[\left(\log \left(\frac{s}{-t}\right)-\mathrm{i} \frac{\pi}{2}\left(1+\kappa_{a b}\right)\right) \mathbf{T}_{t}^{2}+\mathrm{i} \frac{\pi}{2}\left(\mathbf{T}_{s}^{2}-\mathbf{T}_{u}^{2}+\kappa_{a b} \mathbf{T}_{t}^{2}\right)\right]\right\}
$$


The factor $\kappa_{a b}$ distinguishes quark and gluon amplitudes, with $a, b=q, g$, and one has $\kappa_{g g}=\kappa_{q g}=$ 1 , while $\kappa_{q q}=4 / N_{c}^{2}-1$, due to the different symmetry properties of the color factors of the respective amplitudes. $K\left(\alpha_{s}\right)$, on the other hand, is given by a scale integral of the cusp anomalous dimension [34, 35, 36, 37],

$$
K\left(\alpha_{s}\right)=-\frac{1}{4} \int_{0}^{\mu^{2}} \frac{d \lambda^{2}}{\lambda^{2}} \hat{\gamma}_{K}\left(\alpha_{s}\left(\lambda^{2}\right)\right) .
$$

Finally, the interesting feature of the color-singlet operator $\mathscr{Z}_{1, \mathbf{R}}$, whose explicit expression can be found in [11], is that it can be written as a product of factors, each associated to one of the external legs. Distinguishing again quark and gluon scattering one can write

$$
\mathscr{Z}_{\mathbf{1}, \mathbf{R}}^{a b}\left(\frac{t}{\mu^{2}}, \alpha_{s}\right)=\left(\mathscr{Z}_{\mathbf{1}, \mathbf{R}}^{a}\left(\frac{t}{\mu^{2}}, \alpha_{s}\right)\right)^{2}\left(\mathscr{Z}_{\mathbf{1}, \mathbf{R}}^{b}\left(\frac{t}{\mu^{2}}, \alpha_{s}\right)\right)^{2}
$$

making it natural to think of each external leg contribution as a 'jet' factor. These results of infrared factorization at high energy must be compared to the expressions one gets from Regge factorization $[38,39]$, under the common assumptions that the only singularities of these amplitudes in the complex angular momentum plane should be Regge poles. Using these methods one finds that the $t$-channel color octet components of quark and gluon amplitudes can be written as [40,41]

$$
\begin{aligned}
& \mathscr{M}_{a b}^{[8]}\left(\frac{s}{\mu^{2}}, \frac{t}{\mu^{2}}, \alpha_{s}\right)= 2 \pi \alpha_{s} H_{a b}^{(0),[8]} \\
& \times\left\{C_{a}\left(\frac{t}{\mu^{2}}, \alpha_{s}\right)\left[A_{+}\left(\frac{s}{t}, \alpha_{s}\right)+\kappa_{a b} A_{-}\left(\frac{s}{t}, \alpha_{s}\right)\right] C_{\mathrm{b}}\left(\frac{t}{\mu^{2}}, \alpha_{s}\right)\right. \\
&\left.+\mathscr{R}_{a b}^{[8]}\left(\frac{s}{\mu^{2}}, \frac{t}{\mu^{2}}, \alpha_{s}\right)+\mathscr{O}\left(\frac{t}{s}\right)\right\},
\end{aligned}
$$

where the functions $C_{a}$ are impact factors, which depend on the identity of the particles undergoing the high-energy scattering, $H_{a b}^{(0),[8]}$ is the matrix element for tree-level octet exchange, and the (logarithmic) energy dependence is contained in the 'Regge trajectory' factor

$$
A_{ \pm}\left(\frac{s}{t}, \alpha_{s}\right)=\left(\frac{-s}{-t}\right)^{\alpha(t)} \pm\left(\frac{s}{-t}\right)^{\alpha(t)}
$$

We have also included in Eq. (3.5) an octet 'remainder' function $\mathscr{R}_{a b}^{[8]}$, accounting for the fact that Eq. (3.5) is not exact at leading power, but only to leading logarithmic accuracy, and at NLL for the real part of the amplitude [42]. As we will see below, infrared factorization allows us to extract useful information on the remainder, going beyond the contributions of Regge poles.

\section{Comparing factorizations}

The simplest way to compare the two high-energy factorizations, Eq. (2.1) and Eq. (3.5), is to expand the matrix element in powers of the coupling and in powers of the high-energy logarithm, as

$$
\mathscr{M}^{[j]}\left(\frac{s}{\mu^{2}}, \frac{t}{\mu^{2}}, \alpha_{s}\right)=4 \pi \alpha_{s} \sum_{n=0}^{\infty} \sum_{i=0}^{n}\left(\frac{\alpha_{s}}{\pi}\right)^{n} \ln ^{i}\left(\frac{s}{-t}\right) M^{(n), i,[j]}\left(\frac{t}{\mu^{2}}\right),
$$


and perform similar expansion for the various factors on the right-hand sides of Eq. (2.1) and Eq. (3.5). Thus, for any function involving high-energy logarithms, $(n)$ will denote the perturbative order, $i$ the power of $\log (s /|t|)$, and $[j]$ the color representation in a $t$-channel basis. Even before expanding, however, a direct comparison of Eq. (2.1) and Eq. (3.5) at leading logarithmic (LL) level shows that the Regge trajectory $\alpha(t)$ must be strictly related to the cusp integral, Eq. (3.3). More precisely, one finds that if an amplitude, at tree-level, and in the high-energy limit, is dominated by $t$-channel exchange of a particle in representation $[j]$ of the gauge group, then that particle will undergo the process of 'Reggeization', and the divergent contributions to its Regge trajectory will be given by $[9,10,36]$

$$
\alpha^{[j]}\left(\frac{t}{\mu}, \alpha_{s}(\mu)\right)=\mathscr{C}_{2}^{[j]} K\left(\alpha_{s}(-t)\right)
$$

where $\mathscr{C}_{2}^{[j]}$ is the quadratic Casimir eigenvalue of representation $[j]$.

A more detailed analysis allows one to begin identifying the terms that break the Regge-polebased factorization Eq. (3.5), starting at two loops and at NNLL accuracy. Doing this is quite interesting, since at this level a more general picture of Reggeization is expected to arise, involving Regge cuts in the complex angular momentum plane. A precise identification of the contributions to the amplitude which violate Eq. (3.5) may provide key information to jump-start a new kind of high-energy resummation. In this spirit, one observes that at two loops Eq. (2.1) suggests a natural expression for impact factors,

$$
C_{a}^{(2)}=\frac{1}{2} Z_{1, \mathbf{R}, a a}^{(2)}-\frac{1}{8}\left(Z_{1, \mathbf{R}, a a}^{(1)}\right)^{2}+\frac{1}{4} Z_{1, \mathbf{R}, a a}^{(1)} \operatorname{Re}\left[H_{a a}^{(1), 0,[8]} / H_{a a}^{(0),[8]}\right]+\mathscr{O}\left(\varepsilon^{0}\right),
$$

simply arising from the expansion of the 'jet factors' in Eq. (3.4), acting on the finite factors $H^{(n),[8]}$. A direct matching of the two factorizations, however, gives a much more intricate expression for the impact factor, which evidently breaks universality, involving mixing with non-octet components and depending on the identities of all particles involved in the scattering. The expressions derived from IR factorization allow for a precise identification of non-universal terms, which are naturally assigned to the 'remainder' function $\mathscr{R}^{[8]}$. We can check the consistency of our approach by computing the newly defined remainders for quark and gluon amplitudes, which yields double poles of the form

$$
R_{q q}^{(2), 0,[8]}=\frac{\pi^{2}}{4 \varepsilon^{2}}\left(1-\frac{3}{N_{c}^{2}}\right), \quad R_{g g}^{(2), 0,[8]}=-\frac{3 \pi^{2}}{2 \varepsilon^{2}}, \quad R_{q g}^{(2), 0,[8]}=-\frac{\pi^{2}}{4 \varepsilon^{2}} .
$$

Next, we can construct a function measuring the discrepancy between the predictions of Regge factorization for the quark-gluon amplitude, which are based on universality, and the actual matrix elements. We find

$$
\begin{aligned}
\Delta_{(2), 0,[8]} & \equiv \frac{M_{q g}^{(2), 0}}{H_{q g}^{(0),[8]}}-\left[C_{q}^{(2)}+C_{g}^{(2)}+C_{q}^{(1)} C_{g}^{(1)}-\frac{\pi^{2}}{4}\left(1+\kappa_{q g}\right)\left(\alpha^{(1)}\right)^{2}\right] \\
& =\frac{1}{2}\left[R_{q g}^{(2), 0,[8]}-\frac{1}{2}\left(R_{q q}^{(2), 0,[8]}+R_{g g}^{(2), 0,[8]}\right)\right]=\frac{\pi^{2}}{\varepsilon^{2}} \frac{3}{16}\left(\frac{N_{c}^{2}+1}{N_{c}^{2}}\right),
\end{aligned}
$$

which precisely reproduces the result of Ref. [43], where a failure of Eq. (3.5) was first observed. 
Moving on to the three-loops, one finds, as expected, that the breaking of universality occurs at the level of single-logarithmic terms. Indeed, if one attempts to find an expression for the three-loop Regge trajectory using soft-collinear ingredients one finds a set of non-universal terms, involving both color mixing and process-dependent contributions. Following our general strategy, we assign these terms to the remainder functions. This gives a prediction for singular, single-logarithmic terms in three-loop quark and gluon amplitudes, which break Regge universality. As an example, triple pole contributions are given by

$$
R_{q q}^{(3), 1,[8]}=\frac{\pi^{2}}{\varepsilon^{3}} \frac{2 N_{c}^{2}-5}{12 N_{c}}, \quad R_{g g}^{(3), 1,[8]}=-\frac{\pi^{2}}{\varepsilon^{3}} \frac{2}{3} N_{c}, \quad R_{q g}^{(3), 1,[8]}=-\frac{\pi^{2}}{\varepsilon^{3}} \frac{N_{c}}{24} .
$$

Single pole contributions can be computed as well, but they are, as might be expected, considerably lengthier, involving interference with finite two-loop contributions as well.

We conclude this section by noting that, just as infrared factorization provides important information in the high-energy limit, one may also use Regge factorization to extract constraints on the finite parts of the amplitudes, which are not in principle controlled by Eq. (2.1). Specifically, and interestingly, one may show [33] that LL and NLL octet hard parts of quark and gluon amplitudes vanish in dimensional regularization to all orders in perturbation theory. More precisely one finds that

$$
\begin{aligned}
\operatorname{Im}\left(H^{(n), n,[8]}\right) & =0, & \operatorname{Re}\left(H^{(n), n,[8]}\right) & =O\left(\varepsilon^{n}\right), \\
\operatorname{Im}\left(H^{(n), n-1,[8]}\right) & =O\left(\varepsilon^{n}\right), & \operatorname{Re}\left(H^{(n), n-1,[8]}\right) & =\mathscr{O}\left(\varepsilon^{n-2}\right),
\end{aligned}
$$

where the only finite order information used is the vanishing of the one-loop octet hard part. Eq. (4.7) reinforces the idea that high-energy logarithms are infrared in nature: thus, infraredfinite high-energy logarithms must come from the interference of soft and collinear functions with lower-order contributions subleading in $\varepsilon$. These constraints, discussed in greater detail in [33], are the subject of ongoing investigations.

\section{Weaving webs}

Another direction of investigation which has seen remarkable progress in recent years is the development of techniques for the direct calculation of the soft anomalous dimension. The key element of these techniques is the fact that soft gluon contributions to the infrared operator $\mathscr{Z}$ can be expressed in terms of correlators of Wilson lines, and such correlators are known to exponentiate. Furthermore, diagrammatic rules exist that allow one to compute directly the logarithm of the correlator, considerably simplifying the task of extracting the anomalous dimension. For abelian gauge theories, the exponent is very simple, involving only connected diagrams [44], and thus only correlations induced by matter loops. For non-abelian theories, in the case of amplitudes involving only two hard partons, this rule generalizes [45, 46, 47] in a relatively simple way: the exponent is built out of diagrams, called 'webs', which are two-Wilson-line-irreducible, i.e. that cannot be partitioned into disconnected subdiagrams by cutting only the two Wilson lines. These diagrams then enter the exponent with modified color factors which can be recursively computed from the ordinary Feynman rules. The general case of multi-particle non-abelian amplitudes is 
considerably more intricate $[48,49,50,51,52,53]$. In particular, the concept of 'web' must be rather drastically generalized: webs are in this case sets of Feynman diagrams, which are closed under the operation of permuting the order of their attachments to each Wilson line. Writing each diagram $D$ as the product of its color factor $C(D)$ and its kinematic integral $\mathscr{F}(D)$, one finds that each web contributes to the logarithm of the correlator through the combination

$$
W=\sum_{D, D^{\prime} \in W} \mathscr{F}(D) R_{D D^{\prime}} C\left(D^{\prime}\right),
$$

where the sum runs over all diagrams $D, D^{\prime}$ contributing to the web $W$, and $R_{D D^{\prime}}$ is a 'web mixing matrix' whose entries are rational numbers of combinatorial origin.

The physically interesting information in the Wilson line correlator is contained in its ultraviolet divergences, which can be mapped to the infrared divergences of the original scattering amplitude. Extracting these divergences is non-trivial, due to the highly singular nature of these correlators, which contain their own infrared divergences, as well as collinear divergences in the massless case. To disentagle singularities of different physical origin, it is useful to consider nonlightlike Wilson lines (which are of course also directly related to infrared singularities of massive colored particles), and introduce a mass scale $m$ as an infrared regulator. Dimensional regularization then controls the UV singularities which are the target of the calculation. One considers therefore the regulated correlator

$$
\mathscr{S}\left(\gamma_{i j}, \alpha_{s}(\mu), \varepsilon, \frac{m}{\mu}\right) \equiv\left\langle 0\left|\Phi_{\beta_{1}}^{(m)} \otimes \Phi_{\beta_{2}}^{(m)} \otimes \ldots \otimes \Phi_{\beta_{L}}^{(m)}\right| 0\right\rangle,
$$

where $\gamma_{i j}$ are the cusp angles defined in Eq. (2.4), and each Wilson line $\Phi_{\beta_{i}}$, pointing in the direction $\beta_{i}$ defined by the momentum $p_{i}$ of the $i$-th particle, is regulated at large distances by an exponential regulator [12], as

$$
\Phi_{\beta_{i}}^{(m)}=\mathscr{P} \exp \left[\mathrm{i} g \mu^{\varepsilon} \int_{0}^{\infty} d \lambda \beta_{i} \cdot A\left(\lambda \beta_{i}\right) \mathrm{e}^{-m \lambda \sqrt{\beta_{i}^{2}}}\right]
$$

where we take momenta to be time-like, $\beta_{i}^{2}>0$. The multiplicative renormalizability of Wilson line correlators now ensures that a UV finite version of $\mathscr{S}$ can be constructed,

$$
\mathscr{S}_{\text {ren. }}\left(\gamma_{i j}, \alpha_{s}\left(\mu^{2}\right), \varepsilon, \frac{m}{\mu}\right)=\mathscr{S}\left(\gamma_{i j}, \alpha_{s}\left(\mu^{2}\right), \varepsilon, \frac{m}{\mu}\right) Z\left(\gamma_{i j}, \alpha_{s}\left(\mu^{2}\right), \varepsilon\right),
$$

with the matrix renormalization factor $Z$ containing all physically relevant information. The matrix nature of $Z$ entails a further subtlety: in order to properly subtract the UV divergences of $\mathscr{S}$, the expression for $Z$ at high orders must include commutators of lower-order contributions, as dictated by the Baker-Campbell-Haussdorf formula. The structure of these commutator counterterms is easily worked out: for example, up to three loops, one finds that the renormalization factor $Z$ can be expressed in terms of the soft anomalous dimension $\Gamma$ as

$$
\begin{aligned}
Z\left(\alpha_{s}, \varepsilon\right)= & \exp \left\{\alpha_{s} \frac{1}{2 \varepsilon} \Gamma^{(1)}+\alpha_{s}^{2}\left(\frac{1}{4 \varepsilon} \Gamma^{(2)}-\frac{b_{0}}{4 \varepsilon^{2}} \Gamma^{(1)}\right)\right. \\
& \left.+\alpha_{s}^{3}\left(\frac{1}{6 \varepsilon} \Gamma^{(3)}+\frac{1}{48 \varepsilon^{2}}\left[\Gamma^{(1)}, \Gamma^{(2)}\right]-\frac{1}{6 \varepsilon^{2}}\left(b_{0} \Gamma^{(2)}+b_{1} \Gamma^{(1)}\right)+\frac{b_{0}^{2}}{6 \varepsilon^{3}} \Gamma^{(1)}\right)\right\}
\end{aligned}
$$


where $\Gamma^{(n)}$ is the $n$-th order perturbative coefficient of $\Gamma\left(\alpha_{s}\right)$. One sees that in the non-abelian case the logarithm of $Z$ involves multiple UV poles, even in the conformal limit in which the coefficients $b_{n}$ of the $\beta$ function vanish.

Armed with these tools, computing $\Gamma$ is a well defined, if lengthy and rather intricate, task. It is sufficient to compute the regularized soft function $\mathscr{S}$, which can be done directly at the level of the exponent, as

$$
\mathscr{S}\left(\alpha_{s}, \varepsilon\right)=\exp \left[w\left(\alpha_{s}, \varepsilon\right)\right]=\exp \left[\sum_{n=1}^{\infty} \sum_{k=-n}^{\infty} \alpha_{s}^{n} \varepsilon^{k} w^{(n, k)}\right] .
$$

The anomalous dimension is now constructed in terms of combinations of the perturbative coefficients $w^{(n, k)}$, including commutator counterterms. Up to three loops, one gets

$$
\begin{aligned}
& \Gamma^{(1)}=-2 w^{(1,-1)} \\
& \Gamma^{(2)}=-4 w^{(2,-1)}-2\left[w^{(1,-1)}, w^{(1,0)}\right] \\
& \Gamma^{(3)}=-6 w^{(3,-1)}+\frac{3}{2} b_{0}\left[w^{(1,-1)}, w^{(1,1)}\right]+3\left[w^{(1,0)}, w^{(2,-1)}\right]+3\left[w^{(2,0)}, w^{(1,-1)}\right] \\
& +\left[w^{(1,0)},\left[w^{(1,-1)}, w^{(1,0)}\right]\right]-\left[w^{(1,-1)},\left[w^{(1,-1)}, w^{(1,1)}\right]\right] .
\end{aligned}
$$

Notice that, while $\Gamma^{(n)}$ is given, as expected, by a combination of single pole and $\beta$ function contributions, also positive powers of $\varepsilon$ in the logarithm of the regularized correlator play a role. Notice also that the matrix $w\left(\alpha_{s}, \varepsilon\right)$ has a non-trivial structure: order by order, it is the sum of all relevant webs, each of which in general contributes to several color structures. Furthermore, most webs have multiple UV poles, and therefore are not directly physical: they depend on the IR cutoff $m$. Only when appropriate commutator subtractions have been included one is left with a physical contribution to $\Gamma$, corresponding to a single UV pole, and thus independent of $m$.

\section{Multiple gluon exchange webs}

I would like to conclude this contribution by giving a few details about a class of webs which has recently received a lot of attention $[12,13,54]$. These are the webs which are generated if one computes the soft function $\mathscr{S}$ with a path integral weighted by just the quadratic terms in the action

$$
\left.\mathscr{S}\left(\gamma_{i j}, \alpha_{s}(\mu), \varepsilon, \frac{m}{\mu}\right)\right|_{\text {MGEW }} \equiv \int[D A] \Phi_{\beta_{1}}^{(m)} \otimes \Phi_{\beta_{2}}^{(m)} \otimes \ldots \otimes \Phi_{\beta_{L}}^{(m)} \exp \left\{\mathrm{i} S_{0}[A]\right\} .
$$

In diagrammatic terms, these webs involve graphs in which all gluons attach directly to the Wilson lines, and there are no three- or four-gluon vertices: they are called 'Multiple Gluon Exchange Webs' (MGEWs). While their graphical appearance is abelian, it is important to realize that, due to importance of the ordering of gluon insertions on the Wilson lines, they have a true non-abelian character, and they contribute to the same color structures as fully connected webs involving gluon self-interactions.

MGEWs are sufficiently well understood that several of their properties are known or conjectured to all orders in perturbation theory, and in fact, as we will mention below, certain diagrams 
in this class are explicitly known for any number of gluons. To begin with, kinematic factors for diagrams contributing to MGEWs admit a universal parametrization, which leads to an explicit, completely general integral representation. One can write, for any diagram $D$ contributing to a MGEW at $n$ loops,

$$
\mathscr{F}^{(n)}(D)=\kappa^{n} \Gamma(2 n \varepsilon) \int_{0}^{1} \prod_{k=1}^{n}\left[d x_{k} \gamma_{k} P_{\varepsilon}\left(x_{k}, \gamma_{k}\right)\right] \phi_{D}^{(n)}\left(x_{i} ; \varepsilon\right) .
$$

Here $\kappa=-g^{2} /\left(8 \pi^{2}\right)+\mathscr{O}(\varepsilon), \gamma_{k}$ is the cusp angle subtended by the $k$-th gluon, and $x_{k}$ is an anglelike variable measuring the degree of collinearity of the $k$-th gluon with the emitting and the absorbing Wilson lines. $P_{\varepsilon}\left(x_{k}, \gamma_{k}\right)$ is a function arising from the coordinate space gluon propagator, and given by

$$
\left.P_{\varepsilon}(x, \gamma) \equiv\left[x^{2}+(1-x)^{2}-x(1-x) \gamma\right)\right]^{-1+\varepsilon} ;
$$

finally, the kernel $\phi_{D}^{(n)}\left(x_{i} ; \varepsilon\right)$ contains the information about the ordering of gluon attachments on each Wilson line, which is specific to diagram $D$; one may write

$$
\phi_{D}^{(n)}\left(x_{i} ; \varepsilon\right)=\int_{0}^{1} \prod_{k=1}^{n-1} d y_{k}\left(1-y_{k}\right)^{-1+2 \varepsilon} y_{k}^{-1+2 k \varepsilon} \Theta_{D}\left[\left\{x_{k}, y_{k}\right\}\right]
$$

where $\Theta_{D}\left[\left\{x_{k}, y_{k}\right\}\right]$ is a product of Heaviside functions enforcing the proper ordering on each line. The integral representation in Eq. (6.2) is sufficiently general and manageable to allow for calculations at very high orders. To substantiate this, consider the special class of highly symmetric $n$-loop MGE diagrams illustrated in Fig. 1 for $n=6$, which were called 'Escher staircases' in [49]. At each order, there are two such diagrams, enantiomers of each other. As is apparent from their graphical structure, these diagrams have no subdivergences, and thus yield directly a single pole, contributing to the anomalous dimension, without the need for subtractions. Remarkably, these diagrams can be computed for any $n$, and give a very simple result. To display it, define

$$
S_{n}\left(x_{i}\right) \equiv \prod_{i=1}^{n} \frac{1-x_{i}}{x_{i}} .
$$

as well as

$$
\theta_{+}(n) \equiv \theta\left(S_{n}\left(x_{i}\right)-1\right)
$$

and $\theta_{-}(n) \equiv 1-\theta_{+}(n)$, which define the overall orientation of the staircase. In terms of these simple ingredients, one finds that the kernel (6.4) of the staircase diagrams for any $n$ can be written in the remarkably compact form

$$
\phi_{S}^{(n)}\left(x_{i} ; 0\right)=\frac{1}{(n-1) !} \theta_{+}(n)\left(\log \left[S_{n}\left(x_{i}\right)\right]\right)^{n-1} .
$$

This form was used in Ref. [13] to prove an all-order result for a physical contribution to the anomalous dimension: one finds that the coefficient of a specific color structure appearing in the MGEWs which feature the staircase diagrams must vanish for any $n$.

In general, beyond simple and symmetric cases like the staircase diagrams, one must realize that computing the integrals in Eq. (6.2) is only the first step in a more articulate procedure, and 


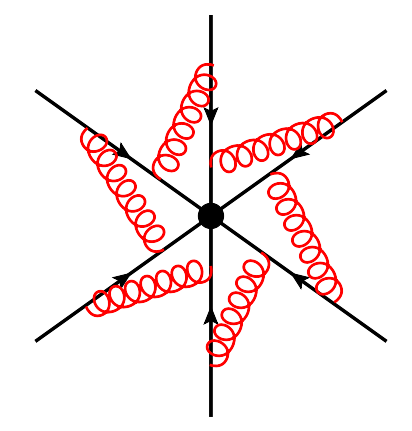

Figure 1: Example of an Escher staircase with six external legs.

several further steps are needed in order to arrive from individual diagrams to the anomalous dimension: diagrams must be combined into webs, which in general comprise several color structures, and, importantly, commutator counterterms must be subtracted. Only at that point multiple poles will cancel, and one can construct contributions to the physical anomalous dimension. Starting from Eq. (6.2), and building up the contribution to the anomalous dimension corresponding to web $W$ and color structure $j$, after subtraction of counterterms, one finds an $\varepsilon$-independent expression of the form

$$
F_{W, j}^{(n)}\left(\alpha_{i}\right)=\int_{0}^{1}\left[\prod_{k=1}^{n} d x_{k} p_{0}\left(x_{k}, \alpha_{k}\right)\right] \mathscr{G}_{W, j}^{(n)}\left(x_{i}, q\left(x_{i}, \alpha_{i}\right)\right),
$$

where the function $p_{0}$ is the limit of Eq. (6.3) as $\varepsilon \rightarrow 0$, now expressed in terms of the variable $\alpha$ defined in Eq. (2.4), while $q(x, \alpha)$ is the logarithm of the quadratic form in $x$ appearing in Eq. (6.3). This logarithm arises when Eq. (6.3) is expanded in powers of $\varepsilon$ and combined with higher-order poles arising in individual diagrams. At this level, and at this level only, the structure of the answer simplifies drastically, and its analytic properties become apparent. One finds, for all webs computed so far, up to four loops and five external legs, that the function $F_{W, j}^{n}$ obeys the following key properties, which were conjectured in [12] to be valid for all MGEWs: first, $F^{n}$ is factorized, that is, it is a sum of terms, each of weight $2 n-1$, and each given by a product of functions depending on individual cusp angles; second, the symbol of $F^{n}$ is built out of a very simple alphabet, comprising only the letters $\left\{\alpha_{i}, 1-\alpha_{i}^{2}\right\}$, with $i=1, \ldots, n$.

These general insights, paired with high-order and all-order examples, suggest that the problem of MGEWs may be solvable in closed form. In parallel, efforts are continuing to compute other, more connected webs contributing to the three-loop soft anomalous dimension for both massless and massive particle scattering. The remarkable progress that has been achieved in the past few years makes it likely that this problem too will be completely solved in the not too far future, a statement that would have been considered quite implausible in previous iterations of this Conference.

\section{Acknowledgements}

Work supported by MIUR (Italy), under contract 2010YJ2NYW_006 and by the University of Torino and the Compagnia di San Paolo under contract ORTO11TPXK. The author thanks Vittorio 
Del Duca, Giulio Falcioni, Einan Gardi, Mark Harley, Chris White and Leonardo Vernazza for the fruitful collaborations that yielded some of the results reviewed here.

\section{References}

[1] See, for example, J. Currie, A. Gehrmann-De Ridder, E. W. N. Glover and J. Pires, JHEP 1401 (2014) 110, [arXiv:1310.3993 [hep-ph]].

[2] G. F. Sterman, In *Boulder 1995, QCD and beyond*, p. 327, [hep-ph/9606312].

[3] L. Magnea, Pramana 72 (2009) 69, [arXiv:0806.3353 [hep-ph]].

[4] E. Gardi and G. Grunberg, Nucl. Phys. B 794 (2008) 61, [arXiv:0709.2877 [hep-ph]].

[5] H. Elvang and Y. -t. Huang, arXiv:1308.1697 [hep-th].

[6] V. Del Duca, C. Duhr and V. A. Smirnov, JHEP 1003 (2010) 099, [arXiv:0911.5332 [hep-ph]].

[7] A. B. Goncharov, M. Spradlin, C. Vergu and A. Volovich, Phys. Rev. Lett. 105 (2010) 151605, [arXiv:1006.5703 [hep-th]].

[8] Z. Bern, L. J. Dixon and V. A. Smirnov, Phys. Rev. D 72 (2005) 085001, [hep-th/0505205].

[9] V. Del Duca, C. Duhr, E. Gardi, L. Magnea and C. D. White, JHEP 1112 (2011) 021, [arXiv:1109.3581 [hep-ph]].

[10] V. Del Duca, C. Duhr, E. Gardi, L. Magnea and C. D. White, Phys. Rev. D 85 (2012) 071104, [arXiv:1108.5947 [hep-ph]].

[11] V. Del Duca, G. Falcioni, L. Magnea and L. Vernazza, Phys. Lett. B 732 (2014) 233, [arXiv:1311.0304 [hep-ph]].

[12] E. Gardi, JHEP 1404 (2014) 044, [arXiv:1310.5268 [hep-ph]].

[13] G. Falcioni, E. Gardi, M. Harley, L. Magnea and C. D. White, arXiv:1407.3477 [hep-ph].

[14] L. J. Dixon, L. Magnea and G. F. Sterman, JHEP 0808 (2008) 022, [arXiv:0805.3515 [hep-ph]].

[15] I. Feige and M. D. Schwartz, arXiv:1403.6472 [hep-ph].

[16] T. Becher and M. Neubert, Phys. Rev. Lett. 102 (2009) 162001, [arXiv:0901.0722 [hep-ph]].

[17] E. Gardi and L. Magnea, Nuovo Cim. C 32N5-6 (2009) 137, [arXiv:0908.3273 [hep-ph]].

[18] L. Magnea and G. F. Sterman, Phys. Rev. D 42 (1990) 4222.

[19] S. M. Aybat, L. J. Dixon and G. F. Sterman, Phys. Rev. Lett. 97 (2006) 072001, [hep-ph/0606254].

[20] S. M. Aybat, L. J. Dixon and G. F. Sterman, Phys. Rev. D 74 (2006) 074004, [hep-ph/0607309].

[21] N. Kidonakis, Phys. Rev. Lett. 102 (2009) 232003, [arXiv:0903.2561 [hep-ph]].

[22] A. Ferroglia, M. Neubert, B. D. Pecjak and L. L. Yang, Phys. Rev. Lett. 103 (2009) 201601, [arXiv:0907.4791 [hep-ph]].

[23] A. Ferroglia, M. Neubert, B. D. Pecjak and L. L. Yang, JHEP 0911 (2009) 062, [arXiv:0908.3676 [hep-ph]].

[24] A. Mitov, G. F. Sterman and I. Sung, Phys. Rev. D 82 (2010) 034020, [arXiv:1005.4646 [hep-ph]]. 
[25] Y. -T. Chien, M. D. Schwartz, D. Simmons-Duffin and I. W. Stewart, Phys. Rev. D 85 (2012) 045010, [arXiv:1109.6010 [hep-th]].

[26] E. Gardi and L. Magnea, JHEP 0903 (2009) 079, [arXiv:0901.1091 [hep-ph].

[27] T. Becher and M. Neubert, JHEP 0906 (2009) 081, [arXiv:0903.1126 [hep-ph]].

[28] G. P. Korchemsky and A. V. Radyushkin, Phys. Lett. B 171 (1986) 459.

[29] G. P. Korchemsky and A. V. Radyushkin, Nucl. Phys. B 283 (1987) 342.

[30] S. Caron-Huot, arXiv:1309.6521 [hep-th].

[31] L. J. Dixon, E. Gardi and L. Magnea, JHEP 1002 (2010) 081, [arXiv:0910.3653 [hep-ph].

[32] V. Ahrens, M. Neubert and L. Vernazza, JHEP 1209 (2012) 138, [arXiv:1208.4847 [hep-ph].

[33] V. Del Duca, G. Falcioni, L. Magnea and L. Vernazza, in preparation.

[34] G. P. Korchemsky, Phys. Lett. B325 (1994) 459, [hep-ph/9311294].

[35] I. A. Korchemskaya, G. P. Korchemsky, Nucl. Phys. B437 (1995) 127, [hep-ph/9409446].

[36] I. A. Korchemskaya, G. P. Korchemsky, Phys. Lett. B387 (1996) 346, [hep-ph/9607229].

[37] L. Magnea, Nucl. Phys. B 593 (2001) 269, [hep-ph/0006255].

[38] P. D. B. Collins, “An Introduction to Regge Theory and High-Energy Physics”, Cambridge 1977.

[39] V. Del Duca, hep-ph/9503226.

[40] I. I. Balitsky, L. N. Lipatov and V. S. Fadin, In *Leningrad 1979, Proceedings, Physics Of Elementary Particles*, Leningrad 1979, 109.

[41] V. S. Fadin and L. N. Lipatov, Nucl. Phys. B 406 (1993) 259.

[42] V. S. Fadin, R. Fiore, M. G. Kozlov and A. V. Reznichenko, Phys. Lett. B 639 (2006) 74, [hep-ph/0602006].

[43] V. Del Duca and E. W. N. Glover, JHEP 0110 (2001) 035, [hep-ph/0109028].

[44] D. R. Yennie, S. C. Frautschi and H. Suura, Annals Phys. 13 (1961) 379.

[45] G. F. Sterman, AIP Conf. Proc. 74 (1981) 22.

[46] J. G. M. Gatheral, Phys. Lett. B 133 (1983) 90.

[47] J. Frenkel and J. C. Taylor, Nucl. Phys. B 246 (1984) 231.

[48] E. Laenen, G. Stavenga and C. D. White, JHEP 0903 (2009) 054 [arXiv:0811.2067 [hep-ph]].

[49] E. Gardi, E. Laenen, G. Stavenga and C. D. White, JHEP 1011 (2010) 155, [arXiv:1008.0098 [hep-ph]].

[50] E. Gardi and C. D. White, JHEP 1103 (2011) 079, [arXiv:1102.0756 [hep-ph]].

[51] E. Gardi, J. M. Smillie and C. D. White, JHEP 1109 (2011) 114, [arXiv:1108.1357 [hep-ph]].

[52] E. Gardi, J. M. Smillie and C. D. White, JHEP 1306 (2013) 088, [arXiv:1304.7040 [hep-ph]].

[53] A. Mitov, G. Sterman and I. Sung, Phys. Rev. D 82 (2010) 096010, [arXiv:1008.0099 [hep-ph]].

[54] J. M. Henn and T. Huber, JHEP 1309 (2013) 147, [arXiv:1304.6418 [hep-th]]. 